\title{
Descriptions of the pupae and larvae of Stenopsyche siamensis Martynov, 1931 (Trichoptera: Stenopsychidae) with notes on larval biology
}

\begin{abstract}
Detailed descriptions are given of the pupae and larvae of Stenopsyche siamensis Martynov, and comparisons are made with the immature stages of other Stenopsyche species previously described from Asia. The larval feeding biology of S. siamensis and its position in stream communities are briefly discussed.
\end{abstract}

Keyword: Pupae; Larvae; Stenopsyche siamensis Martynov 